\title{
COMPLETENESS OF EIGENFUNCTIONS OF STURM-LIOUVILLE PROBLEMS WITH TRANSMISSION CONDITIONS*
}

\author{
AIPING WANG ${ }^{\dagger}$, JIONG SUN $\$$, XIAOLING $\mathrm{HAO}^{\S}$, AND SIQIN YAO§
}

\begin{abstract}
In this paper, we investigate a class of Sturm-Liouville problems with eigenparameterdependent boundary conditions and transmission conditions at an interior point. A self-adjoint linear operator $A$ is defined in a suitable Hilbert space $H$ such that the eigenvalues of such a problem coincide with those of $A$. We show that the operator $A$ has only point spectrum, the eigenvalues of the problem are algebraically simple, and the eigenfunctions of $A$ are complete in $H$.
\end{abstract}

Key words. Eigenparameter-dependent boundary conditions, transmission conditions, eigenvalues, eigenfunctions, completeness.

AMS subject classifications. 34L10, 47E05

Introduction. The Sturm-Liouville theory plays an important role in solving many problems in mathematical physics. It is an active area of research in pure and applied mathematics. In recent years, there has been a growing interest in SturmLiouville problems (SLP's) with eigenparameter-dependent boundary conditions, i.e., the eigenparameter appears not only in the differential equations of the SLP's but also in the boundary conditions of the problems $[6,10,11,16]$. There is a vast amount of literature on this subject (see $[1,2,3,4,5,8,12,14]$, etc.). Moreover, some boundary value problems which may have discontinuities in the solution or its derivative at an interior point $c$ are also studied. Conditions are imposed on the interior point $c$ and such conditions involve left and right limits of solutions and their derivatives at $c$ and are often called "transmission conditions" or "interface conditions". These problems often arise in varied assortment of physical transfer problems.

In this paper, we consider a class of SLP's with eigenparameter-dependent boundary conditions and transmission conditions, i.e., study regular Sturm-Liouville equation

$$
l u:=-\left(a(x) u^{\prime}(x)\right)^{\prime}+q(x) u(x)=\lambda u(x) \quad \text { on } I,
$$

where $I=[-1,0) \cup(0,1], a(x)=a_{1}^{2}$ for $x \in[-1,0)$ and $a(x)=a_{2}^{2}$ for $x \in(0,1], a_{1}, a_{2}$ are nonzero real constants, $q(x) \in L^{1}(I, \mathbb{R})$, and $\lambda \in \mathbb{C}$ is the so-called eigenparameter; with the boundary condition

$$
l_{1} u:=\alpha_{1} u(-1)+\alpha_{2} u^{\prime}(-1)=0,
$$

the eigenparameter-dependent boundary condition

$$
l_{2} u:=\lambda\left(\beta_{1}^{\prime} u(1)-\beta_{2}^{\prime} u^{\prime}(1)\right)+\beta_{1} u(1)-\beta_{2} u^{\prime}(1)=0,
$$

${ }^{*}$ Received November 23, 2007; accepted for publication September 24, 2009. This work was supported by the National Nature Science Foundation of China (10861008), (10901119) and the Doctor Discipline Fund of the Ministry of Education of China (20040126008) and the import talent startup research fund of Tianjin University of Science and Technology (20060433).

${ }^{\dagger}$ Department of Mathematics, Inner Mongolia University, Hohhot 010021, China; and Department of Mathematics, Tianjin University of Science and Technology, Tianjin 300222, China.

$\ddagger$ masun@imu.edu.cn

$\S$ Department of Mathematics, Inner Mongolia University, Hohhot 010021, China. 
and the transmission conditions

$$
\begin{aligned}
& l_{3} u:=u(0+)-\alpha_{3} u(0-)-\beta_{3} u^{\prime}(0-)=0, \\
& l_{4} u:=u^{\prime}(0+)-\alpha_{4} u(0-)-\beta_{4} u^{\prime}(0-)=0,
\end{aligned}
$$

where the coefficients $\alpha_{i}, \beta_{i}, \beta_{j}^{\prime}(i=1, \cdots, 4, j=1,2)$ are real numbers. Throughout this paper, we assume that

$$
\theta=\left|\begin{array}{cc}
\alpha_{3} & \beta_{3} \\
\alpha_{4} & \beta_{4}
\end{array}\right|>0, \quad \rho=\left|\begin{array}{cc}
\beta_{1}^{\prime} & \beta_{1} \\
\beta_{2}^{\prime} & \beta_{2}
\end{array}\right|>0
$$

and $\alpha_{1}^{2}+\alpha_{2}^{2} \neq 0$.

Such research is motivated by the theory of heat and mass transfer, various physical transfer problems (see $[10,13]$ ), vibrating string problems when the string is loaded additionally with point masses(see[17]). Also, some problems with transmission conditions arise in thermal conduction problems for a thin laminated plate (i.e., a plate composed by materials with different characteristics piled in the thickness) [18]. In this class of problems, transmission conditions across the interfaces should be added since the plate is laminated. The study of the structure of the solution in the matching region of the layer with the basis solution in the plate leads to consideration of an eigenvalue problem for a second-order differential operator with piecewise continuous coefficients and transmission conditions.

SLP's with transmission conditions have been studied by many authors. In literature $[1,5,14]$, Mukhtarov et al. gave asymptotic formulas for eigenvalues and the corresponding eigenfunctions for these problems. In [9], the complete descriptions of self-adjoint boundary conditions of the Schrödinger operator with $\delta(x)$ or $\delta^{\prime}(x)$ interaction are given. Adjoint and self-adjoint boundary value problems with interface conditions have been studied by Zettl in [20]. Such problems with point interactions are also studied in [7], etc. In this paper, we also deal with this class of problems by means of that method used by Binding et al. in [2]. We consider a linear operator $A$, which is defined in a suitable Hilbert space $H$ such that the eigenvalues of such a problem coincide with those of $A$, and prove $A$ is a self-adjoint operator. We get that the operator $A$ has only point spectrum, the eigenvalues of the problem are algebraically simple, and the eigenfunctions of $A$ are complete in $H$, i.e. the eigenfunctions form an orthogonal basis. Note that each eigenfunction of $A$ consists of an eigenfunction of the problem and a real number.

In Section 1, a self-adjoint linear operator $A$ is defined in a suitable Hilbert space $H$ such that the eigenvalues of the problem $(0.1)-(0.5)$ coincide with those of $A$. In Section 2, we prove that the eigenvalues of the problem are algebraically simple. Finally, in Section 3, we prove that the operator $A$ has only point spectrum, and the eigenfunctions of $A$ are complete in $H$, they form an orthogonal basis.

1. An operator formulation. The relation between a symmetric linear operator $A$ defined in a suitable Hilbert space $H$ and the problem (0.1)-(0.5) has been introduced in [14]. Here, we repeat the definition and prove that the operator $A$ is self-adjoint, not only symmetric.

We define the inner product in $L^{2}(I)$ as

$$
\langle f, g\rangle_{1}=\frac{1}{a_{1}^{2}} \int_{-1}^{0} f_{1} \bar{g}_{1}+\frac{1}{a_{2}^{2} \theta} \int_{0}^{1} f_{2} \bar{g}_{2}, \quad \forall f, g \in L^{2}(I),
$$


where $f_{1}(x)=\left.f(x)\right|_{[-1,0)}, f_{2}(x)=\left.f(x)\right|_{(0,1]}$. It is easy to verify that $\left(L^{2}(I),\langle\cdot, \cdot\rangle_{1}\right)$ is a Hilbert space. For simplicity, it is denoted by $H_{1}$.

The inner product in $H:=H_{1} \oplus \mathbb{C}$ is defined by

$$
\langle F, G\rangle=\langle f, g\rangle_{1}+\frac{1}{\rho \theta} h \bar{k}
$$

for

$$
F=(f(x), h), \quad G=(g(x), k) \in H,
$$

where $f, g \in H_{1}, h, k \in \mathbb{C}$.

We define the operator $A$ in $H$ as follows:

$$
\begin{gathered}
\mathcal{D}(A)=\left\{(f(x), h) \in H \mid f_{1}, f_{1}^{\prime} \in A C_{l o c}((-1,0)), f_{2}, f_{2}^{\prime} \in A C_{l o c}((0,1)),\right. \\
\left.l f \in H_{1}, l_{1} f=l_{3} f=l_{4} f=0, h=\beta_{1}^{\prime} f(1)-\beta_{2}^{\prime} f^{\prime}(1)\right\}, \\
A F=\left(l f,-\left(\beta_{1} f(1)-\beta_{2} f^{\prime}(1)\right)\right) \quad \text { for } F=\left(f, \beta_{1}^{\prime} f(1)-\beta_{2}^{\prime} f^{\prime}(1)\right) \in \mathcal{D}(A) .
\end{gathered}
$$

Note that by our assumption on $q(x)$ and Theorem 3.2 in [19], for each $(f, h) \in \mathcal{D}(A)$, $f_{1}, f_{1}^{\prime}$ are continuous on $[-1,0]$, and $f_{2}, f_{2}^{\prime}$ are continuous on $[0,1]$. For simplicity, for $(f, h) \in \mathcal{D}(A)$, denote

$$
N(f)=\beta_{1} f(1)-\beta_{2} f^{\prime}(1), \quad N^{\prime}(f)=\beta_{1}^{\prime} f(1)-\beta_{2}^{\prime} f^{\prime}(1) .
$$

So, we can study the problem (0.1)-(0.5) in $H$ by considering the operator equation $A F=\lambda F$. Obviously, we have

Lemma 1.1. The eigenvalues of the boundary value problem (0.1)-(0.5) coincide with those of $A$, and its eigenfunctions are the first components of the corresponding eigenfunctions of $A$.

Lemma 1.2. The domain $\mathcal{D}(A)$ is dense in $H$.

Proof. Suppose that $F \in H$ is orthogonal to all $G \in \mathcal{D}(A)$ with respect to the inner product $\langle\cdot, \cdot\rangle$, where $F=(f(x), h), G=(g(x), k)$. Let $\widetilde{C}_{0}^{\infty}$ denote the set of functions

$$
\phi(x)= \begin{cases}\varphi_{1}(x), & x \in[-1,0) \\ \varphi_{2}(x), & x \in(0,1]\end{cases}
$$

where $\varphi_{1}(x) \in C_{0}^{\infty}[-1,0)$ and $\varphi_{2}(x) \in C_{0}^{\infty}(0,1]$. Since $\widetilde{C}_{0}^{\infty} \oplus 0 \subset \mathcal{D}(A)(0 \in \mathbb{C})$, any $U=(u(x), 0) \in \widetilde{C}_{0}^{\infty} \oplus 0$ is orthogonal to $F$, namely,

$$
\langle F, U\rangle=\frac{1}{a_{1}^{2}} \int_{-1}^{0} f(x) \overline{u(x)} d x+\frac{1}{a_{2}^{2} \theta} \int_{0}^{1} f(x) \overline{u(x)} d x=\langle f, u\rangle_{1} .
$$

This implies that $f(x)$ is orthogonal to $\widetilde{C}_{0}^{\infty}$ in $H_{1}$ and hence vanishes. So, $\langle F, G\rangle=$ $\frac{1}{\rho \theta} h \bar{k}=0$. Thus $h=0$ since $k=N^{\prime}(g)$ can be chosen arbitrarily. So, $F=(0,0)$. Hence, $\mathcal{D}(A)$ is dense in $H$.

TheOREM 1.1. The linear operator $A$ is self-adjoint in $H$. 
Proof. For all $F, G \in \mathcal{D}(A),(0.2)$ implies that $f(-1) \bar{g}^{\prime}(-1)-f^{\prime}(-1) \bar{g}(-1)=0$, and direct calculations using $(0.4)$ and $(0.5)$ then yield that

$$
\begin{aligned}
\langle A F, G\rangle= & \langle F, A G\rangle+W(f, \bar{g} ; 0-)-W(f, \bar{g} ;-1)+\frac{1}{\theta} W(f, \bar{g} ; 1)-\frac{1}{\theta} W(f, \bar{g} ; 0+) \\
& -\frac{1}{\rho \theta}\left(N(f) \overline{N^{\prime}(g)}-N^{\prime}(f) \overline{N(g)}\right)=\langle F, A G\rangle,
\end{aligned}
$$

where $W(f, g ; x)$ denotes the Wronskians $f(x) g^{\prime}(x)-f^{\prime}(x) g(x)$. So, $A$ is symmetric.

It remains to show that if $\langle A F, W\rangle=\langle F, U\rangle$ for all $F=\left(f, N^{\prime}(f)\right) \in \mathcal{D}(A)$, then $W \in \mathcal{D}(A)$ and $A W=U$ (where $W=(w(x), h)$ and $U=(u(x), k)$ ), i.e., (i) $w_{1}, w_{1}^{\prime} \in A C_{l o c}((-1,0)), \quad w_{2}, w_{2}^{\prime} \in A C_{l o c}((0,1))$ and $l w \in H_{1}$; (ii) $h=N^{\prime}(w)=$ $\beta_{1}^{\prime} w(1)-\beta_{2}^{\prime} w^{\prime}(1) ;\left(\right.$ iii) $l_{1} w=l_{3} w=l_{4} w=0$; (iv) $u(x)=l w$; (v) $k=-N(w)=$ $-\beta_{1} w(1)+\beta_{2} w^{\prime}(1)$.

For all $F \in \widetilde{C}_{0}^{\infty} \oplus 0 \subset \mathcal{D}(A)$, we obtain

$$
\frac{1}{a_{1}^{2}} \int_{-1}^{0}(l f) \bar{w} \mathrm{~d} x+\frac{1}{a_{2}^{2} \theta} \int_{0}^{1}(l f) \bar{w} \mathrm{~d} x=\frac{1}{a_{1}^{2}} \int_{-1}^{0} f \bar{u} \mathrm{~d} x+\frac{1}{a_{2}^{2} \theta} \int_{0}^{1} f \bar{u} \mathrm{~d} x,
$$

namely, $\langle l f, w\rangle_{1}=\langle f, u\rangle_{1}$. Hence, by standard Sturm-Liouville theory, (i) and (iv) hold. By (iv), the equation $\langle A F, W\rangle=\langle F, U\rangle, \forall F \in \mathcal{D}(A)$, becomes

$$
\begin{aligned}
& \frac{1}{a_{1}^{2}} \int_{-1}^{0}(l f) \bar{w} \mathrm{~d} x+\frac{1}{a_{2}^{2} \theta} \int_{0}^{1}(l f) \bar{w} \mathrm{~d} x+\frac{-N(f) \bar{h}}{\rho \theta} \\
& =\frac{1}{a_{1}^{2}} \int_{-1}^{0} f(l \bar{w}) \mathrm{d} x+\frac{1}{a_{2}^{2} \theta} \int_{0}^{1} f(l \bar{w}) \mathrm{d} x+\frac{N^{\prime}(f) \bar{k}}{\rho \theta} .
\end{aligned}
$$

So,

$$
\langle l f, w\rangle_{1}=\langle f, l w\rangle_{1}+\frac{N^{\prime}(f) \bar{k}}{\rho \theta}+\frac{N(f) \bar{h}}{\rho \theta} .
$$

However,

$$
\begin{aligned}
\langle l f, w\rangle_{1}= & \frac{1}{a_{1}^{2}} \int_{-1}^{0}\left(-a_{1}^{2} f^{\prime \prime}+q(x) f\right) \bar{w} \mathrm{~d} x+\frac{1}{a_{2}^{2} \theta} \int_{0}^{1}\left(-a_{2}^{2} f^{\prime \prime}+q(x) f\right) \bar{w} \mathrm{~d} x \\
= & \frac{1}{a_{1}^{2}} \int_{-1}^{0} f(l \bar{w}) \mathrm{d} x+\frac{1}{a_{2}^{2} \theta} \int_{0}^{1} f(l \bar{w}) \mathrm{d} x+W(f, \bar{w} ; 0-)-W(f, \bar{w} ;-1)+ \\
& \frac{1}{\theta} W(f, \bar{w} ; 1)-\frac{1}{\theta} W(f, \bar{w} ; 0+) \\
= & \langle f, l w\rangle_{1}+W(f, \bar{w} ; 0-)-W(f, \bar{w} ;-1)+\frac{1}{\theta} W(f, \bar{w} ; 1)-\frac{1}{\theta} W(f, \bar{w} ; 0+) .
\end{aligned}
$$

Hence,

$$
\begin{aligned}
\frac{N^{\prime}(f) \bar{k}}{\rho \theta}+\frac{N(f) \bar{h}}{\rho \theta}= & W(f, \bar{w} ; 0-)-W(f, \bar{w} ;-1)+\frac{1}{\theta} W(f, \bar{w} ; 1)-\frac{1}{\theta} W(f, \bar{w} ; 0+) \\
= & \left(f(0-) \bar{w}^{\prime}(0-)-f^{\prime}(0-) \bar{w}(0-)\right)-\left(f(-1) \bar{w}^{\prime}(-1)-f^{\prime}(-1) \bar{w}(-1)\right) \\
& +\frac{1}{\theta}\left(f(1) \bar{w}^{\prime}(1)-f^{\prime}(1) \bar{w}(1)\right)-\frac{1}{\theta}\left(f(0+) \bar{w}^{\prime}(0+)-f^{\prime}(0+) \bar{w}(0+)\right) .
\end{aligned}
$$


By Naimark's Patching Lemma [15], there is an $F \in \mathcal{D}(A)$ such that $f(-1)=$ $f^{\prime}(-1)=f(0-)=f^{\prime}(0-)=f(0+)=f^{\prime}(0+)=0, f(1)=\beta_{2}^{\prime}$ and $f^{\prime}(1)=\beta_{1}^{\prime}$. For such an $F, N^{\prime}(f)=0$. Thus, from (1.6) we obtain $h=\beta_{1}^{\prime} w(1)-\beta_{2}^{\prime} w^{\prime}(1)$. Namely, (ii) holds. Similarly, one proves (v).

It remains to show that (iii) holds. Choose $F \in \mathcal{D}(A)$ so that $f(1)=f^{\prime}(1)=$ $f(0-)=f^{\prime}(0-)=0, f(-1)=\alpha_{2}$ and $f^{\prime}(-1)=-\alpha_{1}$. Then $N^{\prime}(f)=N(f)=0$. From (1.6), we get $\alpha_{1} w(-1)+\alpha_{2} w^{\prime}(-1)=0$. Let $F \in \mathcal{D}(A)$ satisfies $f(1)=f^{\prime}(1)=$ $f(-1)=f^{\prime}(-1)=f(0+)=0, f(0-)=-\beta_{3}, f^{\prime}(0-)=\alpha_{3}$ and $f^{\prime}(0+)=\theta$. Then $N(f)=N^{\prime}(f)=0$. By (1.6), we have $w(0+)=\alpha_{3} w(0-)+\beta_{3} w^{\prime}(0-)$. Finally, choose $F \in \mathcal{D}(A)$ so that $f(1)=f^{\prime}(1)=f(-1)=f^{\prime}(-1)=f^{\prime}(0+)=0, f(0-)=\beta_{4}$, $f^{\prime}(0-)=-\alpha_{4}$ and $f(0+)=\theta$. Then $N(f)=N^{\prime}(f)=0$. From (1.6), we obtain $w^{\prime}(0+)=\alpha_{4} w(0-)+\beta_{4} w^{\prime}(0-)$.

COROllary 1.1. The eigenvalues of (0.1)-(0.5) are real, and if $\lambda_{1}$ and $\lambda_{2}$ are two different eigenvalues of $(0.1)-(0.5)$, then the corresponding eigenfunctions $f(x)$ and $g(x)$ are orthogonal in the sense of

$$
\frac{1}{a_{1}^{2}} \int_{-1}^{0} f \bar{g}+\frac{1}{a_{2}^{2} \theta} \int_{0}^{1} f \bar{g}+\frac{1}{\rho \theta}\left(\beta_{1}^{\prime} f(1)-\beta_{2}^{\prime} f^{\prime}(1)\right)\left(\beta_{1}^{\prime} \bar{g}(1)-\beta_{2}^{\prime} \bar{g}^{\prime}(1)\right)=0 .
$$

2. The simplicity of eigenvalues. We consider the initial-value problem

$$
\left\{\begin{array}{l}
-a_{1}^{2} u^{\prime \prime}(x)+q(x) u(x)=\lambda u(x), \quad x \in[-1,0], \\
u(-1)=\alpha_{2}, \quad u^{\prime}(-1)=-\alpha_{1} .
\end{array}\right.
$$

In terms of existence and uniqueness in ordinary differential equation theory, the initial-value problem has a unique solution $\varphi_{1}(x, \lambda)$ for every $\lambda \in \mathbb{C}$.

Similarly, the initial-value problem

$$
\left\{\begin{array}{l}
-a_{2}^{2} u^{\prime \prime}(x)+q(x) u(x)=\lambda u(x), \quad x \in[0,1] \\
u(0)=\alpha_{3} \varphi_{1}(0, \lambda)+\beta_{3} \varphi_{1}^{\prime}(0, \lambda) \\
u^{\prime}(0)=\alpha_{4} \varphi_{1}(0, \lambda)+\beta_{4} \varphi_{1}^{\prime}(0, \lambda)
\end{array}\right.
$$

has a unique solution $\varphi_{2}(x, \lambda)$. For each given $x \in[-1,0], \varphi_{1}(x, \lambda)$ is an entire function of $\lambda$; for every $x \in[0,1], \varphi_{2}(x, \lambda)$ is an entire function of $\lambda$. We define a function $\phi(x, \lambda)$ on $x \in[-1,0) \cup(0,1]$ by

$$
\phi(x, \lambda)= \begin{cases}\varphi_{1}(x, \lambda), & x \in[-1,0) \\ \varphi_{2}(x, \lambda), & x \in(0,1]\end{cases}
$$

Obviously, $\phi(x, \lambda)$ satisfies $(0.1),(0.2),(0.4)$ and $(0.5)$

As same as above, we see that the initial-value problem

$$
\left\{\begin{array}{l}
-a_{1}^{2} u^{\prime \prime}(x)+q(x) u(x)=\lambda u(x), \\
u(1)=\beta_{2}{ }^{\prime} \lambda+\beta_{2}, \quad u^{\prime}(1)=\beta_{1}{ }^{\prime} \lambda+\beta_{1} .
\end{array} \quad x \in[0,1],\right.
$$

has a unique solution $\chi_{2}(x, \lambda)$ which is an entire function of the parameter $\lambda$ for each fixed $x \in[0,1]$. And the initial-value problem

$$
\left\{\begin{array}{l}
-a_{2}^{2} u^{\prime \prime}(x)+q(x) u(x)=\lambda u(x), \quad x \in[-1,0], \\
\alpha_{3} u(0)+\beta_{3} u^{\prime}(0)=\chi_{2}(0, \lambda), \\
\alpha_{4} u(0)+\beta_{4} u^{\prime}(0)=\chi_{2}^{\prime}(0, \lambda)
\end{array}\right.
$$


has a unique solution $\chi_{1}(x, \lambda)$ which is an entire function of the parameter $\lambda$ for each fixed $x \in[-1,0]$.

we define the function

$$
\chi(x, \lambda)= \begin{cases}\chi_{1}(x, \lambda), & x \in[-1,0) ; \\ \chi_{2}(x, \lambda), & x \in(0,1],\end{cases}
$$

which satisfies (0.1), (0.3), (0.4) and (0.5).

The Wronskian $W\left(\varphi_{i}(x, \lambda), \chi_{i}(x, \lambda)\right)(i=1,2)$ are independent of the variable $x$. Let $w_{i}(\lambda)=W\left(\varphi_{i}(x, \lambda), \chi_{i}(x, \lambda)\right)$ and $w(\lambda)=w_{1}(\lambda)$, and then we obtain $w_{2}(\lambda)=$ $\theta w(\lambda)$.

Lemma 2.1. The eigenvalues of (0.1)-(0.5) coincide with the zeros of the entire function $w(\lambda)$.

Proof. Let $w\left(\lambda_{0}\right)=0$. Then $W\left(\varphi_{1}\left(x, \lambda_{0}\right), \chi_{1}\left(x, \lambda_{0}\right)\right)=0$ for all $x \in[-1,0]$. Consequently, the functions $\varphi_{1}\left(x, \lambda_{0}\right)$ and $\chi_{1}\left(x, \lambda_{0}\right)$ are linearly dependent; i.e.,

$$
\chi_{1}\left(x, \lambda_{0}\right)=k_{1} \varphi_{1}\left(x, \lambda_{0}\right), \quad x \in[-1,0]
$$

for some $k_{1} \neq 0$. $\mathrm{By}(2.7)$, from this equality we have

$$
\begin{aligned}
\alpha_{1} \chi\left(-1, \lambda_{0}\right)+\alpha_{2} \chi^{\prime}\left(-1, \lambda_{0}\right) & =\alpha_{1} \chi_{1}\left(-1, \lambda_{0}\right)+\alpha_{2} \chi_{1}^{\prime}\left(-1, \lambda_{0}\right) \\
& =k_{1}\left(\alpha_{1} \varphi_{1}\left(-1, \lambda_{0}\right)+\alpha_{2} \varphi_{1}^{\prime}\left(-1, \lambda_{0}\right)\right) \\
& =k_{1}\left(\alpha_{1} \alpha_{2}+\alpha_{2}\left(-\alpha_{1}\right)\right)=0
\end{aligned}
$$

and so $\chi\left(x, \lambda_{0}\right)$ satisfies the first boundary condition. Recalling that the solution $\chi\left(x, \lambda_{0}\right)$ also satisfies the other boundary condition (0.3) and both transmission conditions (0.4) and (0.5) we conclude the $\chi\left(x, \lambda_{0}\right)$ is an eigenfunction of (0.1)-(0.5); i.e., $\lambda_{0}$ is an eigenvalue. Thus, each zero of $w(\lambda)$ is an eigenvalue.

Now let $u_{0}(x)$ be any eigenfunction corresponding to eigenvalue $\lambda_{0}$, but $w\left(\lambda_{0}\right) \neq 0$. Then the function $\varphi_{1}, \chi_{1}$ and $\varphi_{2}, \chi_{2}$ would be linearly independent on $[-1,0]$ and $[0,1]$, respectively. Therefore $u_{0}(x)$ might be represented in the form

$$
u_{0}(x)= \begin{cases}c_{1} \varphi_{1}\left(x, \lambda_{0}\right)+c_{2} \chi_{1}\left(x, \lambda_{0}\right), & x \in[-1,0) \\ c_{3} \varphi_{2}\left(x, \lambda_{0}\right)+c_{4} \chi_{2}\left(x, \lambda_{0}\right), & x \in(0,1]\end{cases}
$$

where at least one of the constants $c_{1}, c_{2}, c_{3}, c_{4}$ where not zero.

Applying the transmission conditions (0.4) and (0.5) to this representation of $u_{0}(x)$ and taking into account the initial conditions (2.8) and (2.10) for $\varphi_{2}\left(x, \lambda_{0}\right)$, and $\chi_{1}\left(x, \lambda_{0}\right)$ respectively, we have

$$
\left\{\begin{array}{l}
\left(c_{1}-c_{3}\right) \varphi_{1}\left(0, \lambda_{0}\right)+\left(c_{2}-c_{4}\right) \chi_{1}\left(0, \lambda_{0}\right)=0 \\
\left(c_{1}-c_{3}\right) \varphi_{1}^{\prime}\left(0, \lambda_{0}\right)+\left(c_{2}-c_{4}\right) \chi_{1}^{\prime}\left(0, \lambda_{0}\right)=0 .
\end{array}\right.
$$

Since

$$
w\left(\lambda_{0}\right)=\left|\begin{array}{ll}
\varphi_{1}\left(0, \lambda_{0}\right) & \chi_{1}\left(0, \lambda_{0}\right) \\
\varphi_{1}^{\prime}\left(0, \lambda_{0}\right) & \chi_{1}^{\prime}\left(0, \lambda_{0}\right)
\end{array}\right| \neq 0
$$

we have $c_{1}-c_{3}=0, \quad c_{2}-c_{4}=0$. 
Now, applying the boundary conditions $(0.2)$ and $(0.3)$ to this representation and taking into account the initial conditions (2.7) and (2.9) for $\varphi_{1}\left(x, \lambda_{0}\right)$ and $\chi_{2}\left(x, \lambda_{0}\right)$, respectively, we have

$$
\begin{aligned}
l_{1}\left(u_{0}(x)\right) & =c_{1} l_{1}\left(\phi\left(x, \lambda_{0}\right)\right)+c_{2} l_{1}\left(\chi\left(x, \lambda_{0}\right)\right) \\
& =c_{1}\left(\alpha_{1} \varphi_{1}\left(-1, \lambda_{0}\right)+\alpha_{2} \varphi_{1}^{\prime}\left(-1, \lambda_{0}\right)\right)+c_{2}\left(\alpha_{1} \chi_{1}\left(-1, \lambda_{0}\right)+\alpha_{2} \chi_{1}^{\prime}\left(-1, \lambda_{0}\right)\right) \\
& =c_{1}\left(\alpha_{1} \alpha_{2}+\alpha_{2}\left(-\alpha_{1}\right)\right)+c_{2}\left(-\varphi_{1}^{\prime}\left(-1, \lambda_{0}\right) \chi_{1}\left(-1, \lambda_{0}\right)+\varphi_{1}\left(-1, \lambda_{0}\right) \chi_{1}^{\prime}\left(-1, \lambda_{0}\right)\right) \\
& =c_{2} w_{1}\left(\lambda_{0}\right)=0 .
\end{aligned}
$$

and

$$
\begin{aligned}
& l_{2}\left(u_{0}(x)\right) \\
& =\left(\lambda_{0} \beta_{1}^{\prime}+\beta_{1}\right)\left(c_{3} \varphi_{2}\left(1, \lambda_{0}\right)+c_{4} \chi_{2}\left(1, \lambda_{0}\right)\right)-\left(\lambda_{0} \beta_{2}^{\prime}+\beta_{2}\right)\left(c_{3} \varphi_{2}^{\prime}\left(1, \lambda_{0}\right)+c_{4} \chi_{2}^{\prime}\left(1, \lambda_{0}\right)\right) \\
& =0
\end{aligned}
$$

we have

$$
\begin{aligned}
& c_{3}\left[\left(\lambda_{0} \beta_{1}^{\prime}+\beta_{1}\right) \varphi_{2}\left(1, \lambda_{0}\right)-\left(\lambda_{0} \beta_{2}^{\prime}+\beta_{2}\right) \varphi_{2}^{\prime}\left(1, \lambda_{0}\right)\right] \\
& \quad+c_{4}\left[\left(\lambda_{0} \beta_{1}^{\prime}+\beta_{1}\right) \chi_{2}\left(1, \lambda_{0}\right)-\left(\lambda_{0} \beta_{2}^{\prime}+\beta_{2}\right) \chi_{2}^{\prime}\left(1, \lambda_{0}\right)\right] \\
& =c_{3}\left(\chi_{2}^{\prime}\left(1, \lambda_{0}\right) \varphi_{2}\left(1, \lambda_{0}\right)-\chi_{2}\left(1, \lambda_{0}\right) \varphi_{2}^{\prime}\left(1, \lambda_{0}\right)\right) \\
& \quad+c_{4}\left[\left(\lambda_{0} \beta_{1}^{\prime}+\beta_{1}\right)\left(\lambda_{0} \beta_{2}^{\prime}+\beta_{2}\right)-\left(\lambda_{0} \beta_{2}^{\prime}+\beta_{2}\right)\left(\lambda_{0} \beta_{1}^{\prime}+\beta_{1}\right)\right] \\
& =c_{3} w_{2}\left(\lambda_{0}\right)=0 .
\end{aligned}
$$

Consequently, $c_{2}=0$ and $c_{3}=0$, since $w_{1}\left(\lambda_{0}\right) \neq 0$ and $w_{2}\left(\lambda_{0}\right) \neq 0$ by assumption. Thus we get he contradiction $c_{1}=c_{2}=c_{3}=c_{4}=0$, which completes the proof.

DefinItion 2.1. The algebraic multiplicity of an eigenvalue $\lambda$ of $(0.1)-(0.5)$ is the order of it as a root of the characteristic equation $w(\lambda)=0$. The geometric multiplicity of an eigenvalue $\lambda$ is the dimension of its eigenspace, i.e., the number of its linearly independent eigenfunctions.

For convenience, set $\phi=\phi(x, \lambda), \chi_{1 \lambda}=\frac{\partial \chi_{1}}{\partial \lambda}, \chi_{1 \lambda}^{\prime}=\frac{\partial \chi_{1}^{\prime}}{\partial \lambda}$, etc.

THEOREM 2.1. The eigenvalues of (0.1)-(0.5) are algebraically simple.

Proof. Let $\lambda=u+i v$. We differentiate the equation $l \chi=\lambda \chi$ with respect to $\lambda$, and have

$$
l \chi_{\lambda}=\lambda \chi_{\lambda}+\chi
$$

By integration by parts, we get

$$
\left\langle l \chi_{\lambda}, \phi\right\rangle_{1}-\left\langle\chi_{\lambda}, l \phi\right\rangle_{1}=\left.\left(\chi_{1 \lambda} \bar{\varphi}_{1}^{\prime}-\chi_{1 \lambda}^{\prime} \bar{\varphi}_{1}\right)\right|_{-1} ^{0}+\left.\frac{1}{\theta}\left(\chi_{2 \lambda} \bar{\varphi}_{2}^{\prime}-\chi_{2 \lambda}^{\prime} \bar{\varphi}_{2}\right)\right|_{0} ^{1} .
$$

Substituting $l \chi_{\lambda}=\lambda \chi_{\lambda}+\chi$ and $l \phi=\lambda \phi$ into the left side of (2.11), we have

$$
\lambda\left\langle\chi_{\lambda}, \phi\right\rangle_{1}+\langle\chi, \phi\rangle_{1}-\left\langle\chi_{\lambda}, \lambda \phi\right\rangle_{1}=\langle\chi, \phi\rangle_{1}+2 i v\left\langle\chi_{\lambda}, \phi\right\rangle_{1} .
$$


Moreover,

$$
\begin{aligned}
& \left.\left(\chi_{1 \lambda} \bar{\varphi}_{1}^{\prime}-\chi_{1 \lambda}^{\prime} \bar{\varphi}_{1}\right)\right|_{-1} ^{0}+\left.\frac{1}{\theta}\left(\chi_{2 \lambda} \bar{\varphi}_{2}^{\prime}-\chi_{2 \lambda}^{\prime} \bar{\varphi}_{2}\right)\right|_{0} ^{1} \\
= & \chi_{1 \lambda}(0, \lambda) \bar{\varphi}_{1}^{\prime}(0, \lambda)-\chi_{1 \lambda}^{\prime}(0, \lambda) \bar{\varphi}_{1}(0, \lambda)-\chi_{1 \lambda}(-1, \lambda) \bar{\varphi}_{1}^{\prime}(-1, \lambda)+\chi_{1 \lambda}^{\prime}(-1, \lambda) \bar{\varphi}_{1}(-1, \lambda) \\
+ & \frac{1}{\theta}\left(\chi_{2 \lambda}(1, \lambda) \bar{\varphi}_{2}^{\prime}(1, \lambda)-\chi_{2 \lambda}^{\prime}(1, \lambda) \bar{\varphi}_{2}(1, \lambda)\right)-\frac{1}{\theta}\left(\chi_{2 \lambda}(0, \lambda) \bar{\varphi}_{2}^{\prime}(0, \lambda)-\chi_{2 \lambda}^{\prime}(0, \lambda) \bar{\varphi}_{2}(0, \lambda)\right) \\
= & \alpha_{1} \chi_{1 \lambda}(-1, \lambda)+\alpha_{2} \chi_{1 \lambda}^{\prime}(-1, \lambda)+\chi_{1 \lambda}(0, \lambda) \bar{\varphi}_{1}^{\prime}(0, \lambda)-\chi_{1 \lambda}^{\prime}(0, \lambda) \bar{\varphi}_{1}(0, \lambda)+ \\
& \frac{1}{\theta}\left(\beta_{2}^{\prime} \bar{\varphi}_{2}^{\prime}(1, \lambda)-\beta_{1}^{\prime} \bar{\varphi}_{2}(1, \lambda)\right)-\frac{1}{\theta}\left(\chi_{2 \lambda}(0, \lambda) \bar{\varphi}_{2}^{\prime}(0, \lambda)-\chi_{2 \lambda}^{\prime}(0, \lambda) \bar{\varphi}_{2}(0, \lambda)\right) \\
= & \alpha_{1} \chi_{1 \lambda}(-1, \lambda)+\alpha_{2} \chi_{1 \lambda}^{\prime}(-1, \lambda)+\frac{1}{\theta}\left(\beta_{2}^{\prime} \bar{\varphi}_{2}^{\prime}(1, \lambda)-\beta_{1}^{\prime} \bar{\varphi}_{2}(1, \lambda)\right) .
\end{aligned}
$$

Note that

$$
w^{\prime}(\lambda)=\alpha_{2} \chi_{1 \lambda}^{\prime}(-1, \lambda)+\alpha_{1} \chi_{1 \lambda}(-1, \lambda)
$$

So, (2.11) becomes

$$
w^{\prime}(\lambda)=\langle\chi, \phi\rangle_{1}+2 i v\left\langle\chi_{\lambda}, \phi\right\rangle_{1}-\frac{1}{\theta}\left(\beta_{2}^{\prime} \bar{\varphi}_{2}^{\prime}(1, \lambda)-\beta_{1}^{\prime} \bar{\varphi}_{2}(1, \lambda)\right) .
$$

Now we consider the simplicity of the eigenvalues of (0.1)-(0.5). Let $\mu$ be arbitrary zero of $w(\lambda)$. By Corollary 1.1, $\mu$ is real. Since

$$
w(\mu)=\left|\begin{array}{ll}
\varphi_{1}(x, \mu) & \chi_{1}(x, \mu) \\
\varphi_{1}^{\prime}(x, \mu) & \chi_{1}^{\prime}(x, \mu)
\end{array}\right|=0
$$

we have $\varphi_{1}(x, \mu)=c_{1} \chi_{1}(x, \mu)\left(c_{1} \neq 0\right)$ and $\varphi_{2}(x, \mu)=c_{2} \chi_{2}(x, \mu)\left(c_{2} \neq 0\right)$, where $c_{1}, c_{2} \in \mathbb{C}$. From

$$
\begin{aligned}
& \varphi_{2}(0, \mu)=c_{1}\left(\alpha_{3} \chi_{1}(0, \mu)+\beta_{3} \chi_{1}^{\prime}(0, \mu)\right)=c_{1} \chi_{2}(0, \mu), \\
& \varphi_{2}^{\prime}(0, \mu)=c_{1}\left(\alpha_{4} \chi_{1}(0, \mu)+\beta_{4} \chi_{1}^{\prime}(0, \mu)\right)=c_{1} \chi_{2}^{\prime}(0, \mu),
\end{aligned}
$$

we get $c_{1}=c_{2} \neq 0$. Thus, simple calculations using (2.12) and the initial values of $\chi_{2}$ at $x=1$ give

$$
w^{\prime}(\mu)=\bar{c}_{1}\left(\frac{1}{a_{1}^{2}} \int_{-1}^{0}\left|\chi_{1}(x, \mu)\right|^{2} \mathrm{~d} x+\frac{1}{a_{2}^{2} \theta} \int_{0}^{1}\left|\chi_{2}(x, \mu)\right|^{2} \mathrm{~d} x+\frac{\rho}{\theta}\right) .
$$

Note that $\rho>0, \theta>0$ and $\bar{c}_{1} \neq 0$, so $w^{\prime}(\mu) \neq 0$. Hence, the algebraic multiplicity of $\mu$ is one. By Lemma 2.1, the proof is completed.

COROllary 2.1. All eigenvalues of (0.1)-(0.5) are also geometrically simple.

Proof. By Lemma 1.1 and Theorem 2.1, we know that the eigenvalues of $A$ are algebraically simple. For the eigenvalue $\lambda$, the problem (0.1)-(0.5) has unique solusion by existence and uniqueness theory of ordinary differential equation. Therefore the conclusion holds. 


\section{Completeness of eigenfunctions.}

Theorem 3.1. The operator $A$ has only point-spectrum, i.e., $\sigma(A)=\sigma_{p}(A)$.

Proof. It suffices to prove that if $\gamma$ is not an eigenvalue of $A$, then $\gamma \in \rho(A)$. Since $A$ is self-adjoint, we only consider real $\gamma$. We investigate the equation $(A-\gamma) Y=$ $F \in H$, where $F=(f, h)$.

Consider the following problem

$$
\left\{\begin{array}{l}
l y-\gamma y=f, \quad x \in I \\
\alpha_{1} y(-1)+\alpha_{2} y^{\prime}(-1)=0 \\
y(0+)=\alpha_{3} y(0-)+\beta_{3} y^{\prime}(0-) \\
y^{\prime}(0+)=\alpha_{4} y(0-)+\beta_{4} y^{\prime}(0-)
\end{array}\right.
$$

Let $u(x)$ be the solution of the equation $l u-\gamma u=0$ satisfying

$$
\begin{gathered}
u(-1)=\alpha_{2}, \quad u^{\prime}(-1)=-\alpha_{1} ; \\
u(0+)=\alpha_{3} u(0-)+\beta_{3} u^{\prime}(0-) ; \\
u^{\prime}(0+)=\alpha_{4} u(0-)+\beta_{4} u^{\prime}(0-) .
\end{gathered}
$$

In fact

$$
u(x)= \begin{cases}u_{1}(x), & x \in[-1,0) \\ u_{2}(x), & x \in(0,1]\end{cases}
$$

where $u_{1}(x)$ is the unique solution of the initial-value problem

$$
\left\{\begin{array}{l}
-a_{1}^{2} u^{\prime \prime}+q(x) u=\gamma u, \quad x \in[-1,0] \\
u(-1)=\alpha_{2}, \quad u^{\prime}(-1)=-\alpha_{1},
\end{array}\right.
$$

and $u_{2}(x)$ is the unique solution of the problem

$$
\left\{\begin{array}{l}
-a_{2}^{2} u^{\prime \prime}+q(x) u=\gamma u, \quad x \in[0,1] \\
u_{2}(0)=\alpha_{3} u_{1}(0)+\beta_{3} u_{1}^{\prime}(0) ; \\
u_{2}^{\prime}(0)=\alpha_{4} u_{1}(0)+\beta_{4} u_{1}^{\prime}(0) .
\end{array}\right.
$$

Let

$$
w(x)= \begin{cases}w_{1}(x), & x \in[-1,0) \\ w_{2}(x), & x \in(0,1]\end{cases}
$$

be a solution of $l w-\gamma w=f$ satisfying

$$
\begin{gathered}
\alpha_{1} w(-1)+\alpha_{2} w^{\prime}(-1)=0 ; \\
w(0+)=\alpha_{3} w(0-)+\beta_{3} w^{\prime}(0-) ; \\
w^{\prime}(0+)=\alpha_{4} w(0-)+\beta_{4} w^{\prime}(0-) .
\end{gathered}
$$


Then (3.13) has the general solution

$$
y(x)= \begin{cases}d u_{1}+w_{1}, & x \in[-1,0) \\ d u_{2}+w_{2}, & x \in(0,1]\end{cases}
$$

where $d \in \mathbb{C}$.

By the fact that $\gamma$ is not an eigenvalue of $(0.1)-(0.5)$, we have

$$
\gamma\left(\beta_{1}^{\prime} u_{2}(1)-\beta_{2}^{\prime} u_{2}^{\prime}(1)\right)+\left(\beta_{1} u_{2}(1)-\beta_{2} u_{2}^{\prime}(1)\right) \neq 0 .
$$

The second component of $(A-\gamma) Y=F$ involves the equation

$$
-N(y)-\gamma N^{\prime}(y)=h
$$

namely,

$$
\beta_{2} y^{\prime}(1)-\beta_{1} y(1)-\gamma\left(\beta_{1}^{\prime} y(1)-\beta_{2}^{\prime} y^{\prime}(1)\right)=h .
$$

Substituting (3.15) into (3.17), we get

$$
\begin{aligned}
& \left(\beta_{2} u_{2}^{\prime}(1)-\beta_{1} u_{2}(1)+\gamma \beta_{2}^{\prime} u_{2}^{\prime}(1)-\gamma \beta_{1}^{\prime} u_{2}(1)\right) d \\
& =h+\beta_{1} w_{2}(1)-\beta_{2} w_{2}^{\prime}(1)+\gamma \beta_{1}^{\prime} w_{2}(1)-\gamma \beta_{2}^{\prime} w_{2}^{\prime}(1) .
\end{aligned}
$$

In view of (3.16), we know that $d$ is uniquely solvable. Hence, $y$ is uniquely determined.

The above arguments show that $(A-\gamma I)^{-1}$ is defined on all of $H$. We obtain that $(A-\gamma I)^{-1}$ is bounded by Theorem 1.1 and the closed graph theorem. Thus, $\gamma \in \rho(A)$. Hence, $\sigma(A)=\sigma_{p}(A)$.

Using similar method of [1], we have the following Lemma:

Lemma 3.1. The eigenvalues of the boundary value problem (0.1)-(0.5) are bounded below, and they are countably infinite and can cluster only at $\infty$.

Proof. Without loss generalization we assume $\beta_{2}^{\prime} \neq 0$, and $a(x)=1$. And $\varphi_{1}(x, \lambda), \varphi_{2}(x, \lambda), \chi_{1}(x, \lambda), \chi_{2}(x, \lambda)$ are defined in (2.7)-(2.10). First we prove the following conclusion :

$$
\begin{aligned}
& \chi_{1}(x, \lambda)=-s^{3} \beta_{2}^{\prime} \frac{\beta_{3}}{\rho} \sin s \cos [s(-x)]+O\left(|s|^{2} e^{|t|(-x)}\right), \\
& \chi_{1}^{\prime}(x, \lambda)=-s^{4} \beta_{2}^{\prime} \frac{\beta_{3}}{\rho} \sin s \sin [s(-x)]+O\left(|s|^{3} e^{|t|(-x)}\right) .
\end{aligned}
$$

as $|\lambda| \rightarrow \infty$, where $\lambda=s^{2}, s=\sigma+i t, \beta_{2}^{\prime} \neq 0$. These asymptotic formulae uniformly hold for $x$.

By the method of variation of parameters, we have $\chi_{2}(x, \lambda)$ satisfies the following integral equations

$$
\begin{aligned}
\chi_{2}(x, \lambda)= & \left(\beta_{2}+s^{2} \beta_{2}^{\prime}\right) \cos [s(1-x)]+\frac{1}{s}\left(\beta_{1}+s^{2} \beta_{1}^{\prime}\right) \sin [s(1-x)] \\
& +\frac{1}{s} \int_{x}^{1} \sin [s(y-x)] q(y) \chi_{2}(y, \lambda) d y .
\end{aligned}
$$

Let $\chi_{2}(x, \lambda)=|s|^{2} e^{|t|(1-x)} F_{2}(x, \lambda)$, then

$$
\begin{aligned}
F_{2}(x, \lambda)= & {\left[\left(\beta_{2}+s^{2} \beta_{2}^{\prime}\right) \cos [s(1-x)]+\frac{1}{s}\left(\beta_{1}+s^{2} \beta_{1}^{\prime}\right) \sin [s(1-x)]\right]|s|^{-2} e^{-|t|(1-x)} } \\
& +\frac{1}{s} \int_{x}^{1} \sin [s(y-x)] e^{-|t|(y-x)} q(y) F_{2}(y, \lambda) d y .
\end{aligned}
$$


Denote $\mu_{2}(\lambda):=\max _{0 \leq x \leq 1}\left|F_{2}(x, \lambda)\right|$, from the last equation we get that

$$
\mu_{2}(\lambda) \leq\left|\beta_{2}^{\prime}\right|+\frac{M_{2}}{|s|}
$$

for some $M_{2}>0$. Consequently $\mu(\lambda)=O(1)$ as $|\lambda| \rightarrow \infty$, we have

$$
\chi_{2}(x, \lambda)=|s|^{2} e^{|t|(1-x)} F_{2}(x, \lambda)=O\left(|s|^{2} e^{|t|(1-x)}\right) .
$$

Substituting(3.20)in(3.19), we get

$$
\begin{aligned}
\chi_{2}(x, \lambda) & =\left(\beta_{2}+s^{2} \beta_{2}^{\prime}\right) \cos [s(1-x)]+\frac{1}{s}\left(\beta_{1}+s^{2} \beta_{1}^{\prime}\right) \sin [s(1-x)] \\
& +\frac{1}{s} \int_{x}^{1} \sin [s(y-x)] q(y) O\left(|s|^{2} e^{|t|(1-y)}\right) d y \\
& =s^{2} \beta_{2}^{\prime} \cos [s(1-x)]+O\left(|s| e^{|t|(1-x)}\right) .
\end{aligned}
$$

So the asymptotic approximation of $\chi_{2}(x, \lambda)$ is

$$
\chi_{2}(x, \lambda)=s^{2} \beta_{2}^{\prime} \cos [s(1-x)]+O\left(|s| e^{|t|(1-x)}\right) .
$$

We differentiate the equation (3.21) with respect to $\lambda$, and substituting(3.20), we have

$$
\begin{aligned}
\chi_{2 \lambda}^{\prime}(x) & =s\left(\beta_{2}+s^{2} \beta_{2}^{\prime}\right) \sin [s(1-x)]-\left(\beta_{1}+s^{2} \beta_{1}^{\prime}\right) \cos [s(1-x)] \\
& -\int_{x}^{1} \cos [s(y-x)] q(y) O\left(|s|^{2} e^{|t|(1-y)}\right) d y \\
& =s^{3} \beta_{2}^{\prime} \sin [s(1-x)]+O\left(|s|^{2} e^{|t|(1-x)}\right)
\end{aligned}
$$

consequently we have

$$
\chi_{2 \lambda}^{\prime}=s^{3} \beta_{2}^{\prime} \sin [s(1-x)]+O\left(|s|^{2} e^{|t|(1-x)}\right),
$$

Now we study asymptotic approximations of $\chi_{1}(x, \lambda)$ and $\chi_{1}^{\prime}(x, \lambda)$. By the method of variation of parameters, we have $\chi_{1}(x, \lambda)$ satisfies the following integral equation:

$$
\begin{aligned}
\chi_{1}(x, \lambda)= & \frac{1}{\theta}\left(\beta_{4} \chi_{2}(0, \lambda)-\beta_{3} \chi_{2}^{\prime}(0, \lambda)\right) \cos [s(-x)] \\
& +\frac{1}{\theta s}\left(-\alpha_{4} \chi_{2}(0, \lambda)+\alpha_{3} \chi_{2}^{\prime}(0, \lambda)\right) \sin [s(-x)] \\
& +\frac{1}{s} \int_{x}^{0} \sin [s(y-x)] q(y) \chi_{1 \lambda}(y) d y .
\end{aligned}
$$

Substituting(3.21), (3.22) in above equation, then we get

$$
\begin{aligned}
\chi_{1}(x, \lambda)= & -s^{3} \beta_{2}^{\prime} \frac{\beta_{3}}{\theta} \sin s \cos [s(-x)] \\
& +s^{2} \beta_{2}^{\prime}\left(\frac{\beta_{4}}{\theta} \cos s \cos [s(-x)]+\frac{\alpha_{3}}{\theta} \sin s \sin [s(-x)]\right) \\
& -s \beta_{2}^{\prime} \frac{\alpha_{4}}{\theta} \cos s \sin [s(-x)] \\
& +\frac{1}{s} \int_{x}^{0} \sin [s(y-x)] q(y) \chi_{1}(y, \lambda) d y+O\left(|s|^{2} e^{|t|}\right) .
\end{aligned}
$$


Let $\chi_{1}(x, \lambda)=|s|^{3} e^{|t|(-x)} F_{1}(x, \lambda)$, then

$$
\begin{aligned}
F_{1}(x, \lambda)= & -s^{3} \beta_{2}^{\prime} \frac{\beta_{3}}{\theta} \sin s \cos [s(-x)] \\
& +s^{2} \beta_{2}^{\prime}\left(\frac{\beta_{4}}{\theta} \cos s \cos [s(0-x)]+\frac{\alpha_{3}}{\theta} \sin s \sin [s(-x)]\right) \\
& \left.-s \beta_{2}^{\prime} \frac{\alpha_{4}}{\theta} \cos s \sin [s(-x)]\right]|s|^{-3} e^{-|t|(-x)} \\
& +\frac{1}{s} \int_{x}^{0} \sin [s(y-x)] e^{-|t|(-x)} q(y) F_{1}(y, \lambda) d y+O\left(|s|^{-1} e^{|t|}\right) .
\end{aligned}
$$

Denote $\mu_{1}(\lambda)=\max _{-1 \leq x \leq 0}\left|F_{1}(x, \lambda)\right|$, from the last equation we have that

$$
\mu_{1}(\lambda) \leq\left|\beta_{2}^{\prime} \frac{\beta_{3}}{\theta}\right|+\frac{M_{1}}{|s|}
$$

for some $M_{1}>0$. Consequently $\mu_{1}(\lambda)=O(1)$. So when $|\lambda| \rightarrow \infty$, we have

$$
\chi_{1}(x, \lambda)=O\left(|s|^{3} e^{|t|(-x)}\right) .
$$

Substituting(3.24) in (3.23), we get

$$
\begin{aligned}
\chi_{1}(x, \lambda) & =-s^{3} \beta_{2}^{\prime} \frac{\beta_{3}}{\theta} \sin s \cos [s(-x)] \\
& +s^{2} \beta_{2}^{\prime}\left(\frac{\beta_{4}}{\theta} \cos s \cos [s(-x)]+\frac{\alpha_{3}}{\theta} \sin s \sin [s(-x)]\right) \\
& -s \beta_{2}^{\prime} \frac{\alpha_{4}}{\theta} \cos s \sin [s(-x)] \\
& +\frac{1}{s} \int_{x}^{0} \sin [s(y-x)] q(y) O\left(|s|^{3} e^{|t|(-y)}\right) d y+O\left(|s|^{2} e^{|t|}\right) \\
& =-s^{3} \beta_{2}^{\prime} \frac{\beta_{3}}{\theta} \sin s \cos [s(-x)]+O\left(|s|^{2} e^{|t|(-x)}\right),
\end{aligned}
$$

consequently we have

$$
\chi_{1}(x, \lambda)=-s^{3} \beta_{2}^{\prime} \frac{\beta_{3}}{\theta} \sin s \cos [s(-x)]+O\left(|s|^{2} e^{|t|(-x)}\right) .
$$

We differentiate the equation (3.25) with respect to $\lambda$, and substituting (3.24), we get

$$
\chi_{1}^{\prime}(x, \lambda)=-s^{4} \beta_{2}^{\prime} \frac{\beta_{3}}{\theta} \sin s \sin [s(-x)]+O\left(|s|^{3} e^{|t|(-x)}\right) .
$$

By Lemma 2.1, we know the eigenvalues of (0.1)-(0.5)coincide with the zeros of the following entire function

$$
\begin{aligned}
w(\lambda) & =\left|\begin{array}{cc}
\alpha_{2} & \chi_{1}(-1, \lambda) \\
-\alpha_{1} & \chi_{1}^{\prime}(-1, \lambda)
\end{array}\right| \\
& =-s^{4} \beta_{2}^{\prime} \alpha_{2} \frac{\beta_{3}}{\theta} \sin s \sin s+O\left(|s|^{3} e^{|t|}\right) .
\end{aligned}
$$

Denote $w^{*}(\lambda)=-s^{4} \beta_{2}^{\prime} \alpha_{2} \frac{\beta_{3}}{\theta} \sin s \sin s, \alpha(\lambda)=O\left(|s|^{3} e^{|t|}\right)$. Let the closed curve $\Gamma_{n}=\Gamma_{n}^{\prime} \cup \Gamma_{n}^{\prime \prime}(n=1,2, \cdots)$ on plane of $\lambda$ :

$$
\Gamma_{n}^{\prime}=\left\{\lambda=s^{2}=(\sigma+i t)^{2}|| \sigma \mid=\left(n+\frac{1}{2}\right) \pi, 0 \leq t \leq n\right\},
$$




$$
\Gamma_{n}^{\prime \prime}=\left\{\lambda=s^{2}=(\sigma+i t)^{2}|| \sigma \mid \leq\left(n+\frac{1}{2}\right) \pi, t=n\right\} .
$$

On $\Gamma_{n}^{\prime}$, we have

$$
|\sin s|=\frac{1}{2}\left|i e^{-t}+i e^{t}\right|=\frac{e^{t}}{2}\left[1+e^{-2 t}\right]>\frac{e^{|t|}}{4},
$$

On $\Gamma_{n}^{\prime \prime}$, we have

$$
\begin{aligned}
|\sin s| & =\frac{1}{2}\left|e^{i \sigma} e^{-n}-e^{i \sigma} e^{n}\right| \\
& =\frac{e^{n}}{2}\left[1-e^{2 i \sigma} e^{-2 n}\right]>\frac{e^{n}}{4} .
\end{aligned}
$$

Consequently,

$$
\begin{aligned}
\left|w^{*}(\lambda)\right|_{\Gamma_{n}} & =\left|-s^{4} \beta_{2}^{\prime} \alpha_{2} \frac{\beta_{3}}{\theta} \sin s \sin s\right|_{\Gamma_{n}} \\
& >\frac{n}{4} e^{|t|}\left|s^{3} \beta_{2}^{\prime} \alpha_{2} \frac{\beta_{3}}{\theta} \sin s\right| .
\end{aligned}
$$

So when $n$ is big enough, we have

$$
\left|w^{*}(\lambda)\right|_{\Gamma_{n}}>|\alpha(\lambda)|_{\Gamma_{n}} .
$$

By Rouche Theorem $w(\lambda)$ and $w^{*}(\lambda)$ have the same number of zeros interior of $\Gamma_{n}$. Yet $w^{*}(\lambda)$ have $n+1$ zeros interior of $\Gamma_{n}$ as

$$
0, \pi^{2},(2 \pi)^{2}, \cdots,(n \pi)^{2} .
$$

Therefor $w(\lambda)$ have and only have $n+1$ zeros interior of $\Gamma_{n}$. On the other hand, let $s=i t$, viz. $\lambda=-t^{2}$, then we can prove $\left|w^{*}(\lambda)\right|>|\alpha(\lambda)|$, as $|t| \rightarrow \infty$, as same as above, it shows $w\left(-t^{2}\right) \neq 0$ when $t$ is big enough. Consequently, $w(\lambda)$ only have finite negative zeros.

When $\beta_{2}^{\prime}=0$, or $a(x) \neq 1$, we have similar asymptotic formulae, the results hold.

By Theorem 1.1, Theorem 3.1, Lemma 3.1 and the spectral theorem for compact operator, we obtain the following theorem.

THEOREM 3.2. The all eigenfunctions of the problem (0.1)-(0.5), augmented to eigenfunctions of $A$, form an orthonormal basis in $H$, i.e., the eigenfunctions of $A$ are complete in $H$. If let $\left\{\Phi_{n}=\left(\phi_{n}(x), N^{\prime}\left(\phi_{n}\right)\right) ; n \in \mathbb{N}\right\}$ be all orthonormal eigenfunctions of $A$, where $\left\{\phi_{n}(x) ; n \in \mathbb{N}\right\}$ are eigenfunctions of (0.1)-(0.5), then for all $F \in H, F=\sum_{n=1}^{\infty}\left\langle F, \Phi_{n}\right\rangle \Phi_{n}$.

Proof. For every $\delta \in \mathbb{R} \backslash \sigma_{p}(A)$, we know that if $\lambda$ be an eigenvalue of $A-\delta I$, and $V$ a corresponding eigenfunction, then $\frac{1}{\lambda}$ is an eigenvalue of $(A-\delta I)^{-1}$, and $V$ is a corresponding eigenfunction, the converse is also true. By Lemma 3.1 we may assume that

$$
\left|\lambda_{1}-\delta\right| \leq\left|\lambda_{2}-\delta\right| \leq \cdots \leq\left|\lambda_{n}-\delta\right| \leq \cdots \rightarrow \infty .
$$

Let $\left\{\mu_{n} ; n \in \mathbb{N}\right\}$ be the sequence of eigenvalues of $(A-\delta I)^{-1}$. Then, $\mu_{n}=\frac{1}{\lambda_{n}-\delta}$ and

$$
\left|\mu_{1}\right| \geq\left|\mu_{2}\right| \geq \cdots \geq\left|\mu_{n}\right| \geq \cdots \rightarrow 0 .
$$


Note that, 0 is not an eigenvalue of $(A-\delta I)^{-1}$, the dimension of the eigenspace is finite, we obtain the operator $A$ has compact resolvent, i.e., for each $\delta \in \mathbb{R} \backslash \sigma(A)=$ $\mathbb{R} \backslash \sigma_{p}(A),(A-\delta I)^{-1}$ is compact on $H$. By the spectral theory of compact operator, the conclusion hold.

The authors thank referees for their very useful comments and suggestions.

\section{REFERENCES}

[1] N. Altinisik, M.Kadakal and O. Sh. Mukhtarov, Eigenvalues and eigenfunctions of discontinuous Sturm-Liouville problems with eigenparameter-dependent boundary conditions, Acta Math. Hungar., 102 (2004), no. 1-2, pp. 159-175.

[2] P. A. Binding, P. J. Browne And B. A. WAtson, Sturm-Liouville problems with boundary conditions rationally dependent on the eigenparameter, II, Journal of Computational and Applied Mathematics, 148 (2002), pp. 147-168.

[3] P. A. Binding And P. J. Browne, Oscillation theory for indefinite Sturm-Liouville problems with eigenparameter dependent boundary conditions, Proc. Roy. Soc. Edinburg, 127A (1997), pp. 1123-1136.

[4] P. Binding, R. HRyniv And H. LANGer, Elliptic eigenvalue problems with eigenparameter dependent boundary conditions, Journal of Differential Equations, 174 (2001), pp. 30-54.

[5] M. Demirci, Z. AkdoĞan And O. Sh. Mukhtarov, Asymptotic behavior of eigenvalues and eigenfunctions of one discontinuous boundary-value problem, International Journal of Computational Cognition, 2:3 (2004), pp. 101-113.

[6] C. T. Fulton, Two-point boundary value problems with eigenvalue parameter contained in the boundary conditions, Proceedings of the Royal Society of Edinburgh, 77A (1977), pp. 293308.

[7] F. GESZTESy AND W. KIRSCH, One-dimensional Schrödinger operators with interactions on a discerete set, J. fuer Mathematic, 362 (1985), pp. 28-50.

[8] D. B. Hinton, An expansion theorem for an eigenvalue problem with eigenvalue parameter in the boundary condition, Quart. J. Math, Oxford, 30 (1979), pp. 33-42.

[9] Y. S. LI, J. SUN AND Z. WANG, On the complete description of self-adjoint boundary conditions of the Schrödinger operator with a $\delta(x)$ or $\delta^{\prime}(x)$ interaction, Symposium of the fifth Conference of Mathematics Society of Inner Mongolia, Inner Mongolia University Press, 1995, pp. 27-30.

[10] A. V. LyKov And And Yu. A. MikhaíLov, The theory of heat and mass transfer, translated from Russian by I. Shechtman, Israel Program for Scientific Translations, Jerusalem, 1965.

[11] R. Mennicken And M. Möller, Non-self-adjoint boundary eigenvalue problem, North-Holland Mathematics Studies, 192, North-Hplland Publishing Co., Amsterdan, 2003.

[12] O. Sh. Mukhtarov, Discontinuous boundary-value problem with spectral parameter in boundary conditions, Turkish Journal of Mathematics, 18 (1994), pp. 183-192.

[13] O. Sh. Mukhtarov and S. Yakubov, Problems for differential equations with transmission conditions, Applicable Analysis, 81 (2002), pp. 1033-1064.

[14] O. Sh. Mukhtarov and M. Kadakal, Spectral properties of of one Sturm-Liouville type problem with discoutinous weight, (Russian) Sibirsk. MAth. Zh., 46:4 (2005), pp. 860-785; translation in Siberian Math. J., 46:4 (2005), pp. 681-694.

[15] M. A. NAimark, Linear differential operators, part II, Harrap, London, 1968.

[16] M. L. Rasulov, Matheds of contour integration. Series in Applied Mathematics and Mechanics, Vol.3, North-Holland Publishing Co. Amsterdam; Interscience Publishers John Wiley \& Sons, Inc. New york 1967.

[17] A. N. Tikhonov and A. A. Samarskit, Equations of Mathematical Physics, Dover Publications, 1990.

[18] I. TiteuX And Y. Yakubov, Completeness of root functions for thermal conduction in a strip with piecewise continuous coefficients, Math. Models Methods Appl. Sc., 7:7 (1997), pp. $1035-1050$.

[19] J. Weidmann, Spectral theory of ordinary differential operators, Lecture Notes in Math. 1258, Springer-Verlag, Berline, 1987.

[20] A. ZetTL, Adjoint and self-adjoint boundary value problems with interface conditions, SIAM J. Applied Math., 16 (1968), pp. 851-859. 